\title{
Epidemiology and outcome of lymphomas in HIV-infected patients: a multicenter retrospective study
}

\begin{abstract}
Marina Popova ${ }^{1}$, Tatyana Shneyder ${ }^{2}$, Igor Karaygin ${ }^{2}$, Olga Ponomarenko ${ }^{2}$, Ilya Zuyzgin ${ }^{2,11}$, Olga Ryabykina ${ }^{2}$, Olga Ruzhinskaya $^{2}$, Olga Uspenskaya ${ }^{2}$, Nadezhda Medvedeva ${ }^{3}$, Anna Klimovich ${ }^{3}$, Vsevolod Potapenko ${ }^{3}$, Natalya Kotova ${ }^{3,11}$, Elena Zinina $^{4}$, Natalya Popova ${ }^{4}$, Ylia Zhurba ${ }^{4}$, Alexander Myasnikov ${ }^{5}$, Svetlana Moshnina ${ }^{5}$, Alexey Evseev ${ }^{5}$, Tatyana Pospelova ${ }^{6}, K^{2}-$ mil Kaplanov ${ }^{7}$, Tatyana Ksenzova ${ }^{8}$, Elena Karyagina ${ }^{9}$, Zhanna Stolypina ${ }^{9}$, Svetlana Dzola ${ }^{10}$, Alexander Levanov ${ }^{10}$, Evgenya Borzenkova $^{1}$, Anastasya Nekrasova ${ }^{1}$, Natalya Mikhaylova ${ }^{1}$, Ludmila Zubarovskaya ${ }^{1}$, Boris Afanasyev ${ }^{1}$
\end{abstract}

${ }^{1}$ Raisa Gorbacheva Institute of Children Oncology, Hematology and Transplantation, First Pavlov State Medical University of St.Petersburg;

${ }^{2}$ Leningrad Regional Clinical Hospital, St.Petersburg;

${ }^{3}$ City Clinical Hospital №31, St.Petersburg;

${ }^{4}$ Surgut District Clinical Hospital, Surgut;

${ }^{5}$ Republican Clinical Hospital, Petrozavodsk;

${ }^{6}$ Center of Hematology, Novosibirsk;

Contact: Dr. Marina O.Popova

E-mail: marina.popova.spb@gmail.com

\section{Background}

Since the beginning of global epidemic, almost 70 million of people have been infected with the HIV virus, and about 35 million people have died of AIDS. HIV infected patients are at risk for cancer including lymphomas, despite the widespread accessibility of highly active antiretroviral therapy (HAART). In parallel with increasing number of patients living with HIV, the number of patients suffering from HIV-associated malignancies of hematopoietic and lymphoid tissues has increased as well. The aim of our study was to determine epidemiological characteristics and the outcome of lymphomas in HIV-infected patients in Russian Federation.

\section{Materials and methods}

We performed a retrospective multicenter study. The inclusion criterion was a diagnosis of lymphoma in HIV-infected patients. Seventy-three patients were enrolled over a period from May 2006 to Dec 2015. We analyzed data of medical histories, laboratory tests, treatment in hematological hospitals and AIDS Centers, as based on established local guidelines. The median follow-up of the patients was $24(2-110)$ months.
${ }^{7}$ Volgograd Regional Clinical Oncologycal Hospital, Volgograd;

${ }^{8}$ Regional Clinical Hospital, Tumen';

${ }^{9}$ City Clinical Hospital №15, St.Petersburg;

${ }^{10}$ Clinic of Profpathology and Hematology, Saratov State Medical University, Saratov;

${ }^{11}$ Petrov Research Institute of Oncology, St.Petersburg

\section{Results}

Epidemiological and clinical characteristics (diagnosis) of the patients' are presented in Figure 1. Table 1 presents clinical features of the HIV patients with lymphoproliferative disorders, characteristics of lymphoma status, and treatment data. Overall survival at 2 years in HIV-infected patients with lymphomas was 64\% (HL, 80\%; NHL, 60\%; MM, 100\%), time to progression (TTP) was 17\% (HL, 20\%; NHL, 16.7\%; MM, 0\%). Favorable prognostic factors for OS were: chemotherapy (ChT) in combination with HAART, adequacy of ChT for the type and stage of lymphoma. Favorable prognostic factors for TTP were: adequate ChT for the type and stage of disorder. Adverse prognostic factors for OS and TTP were: $\mathrm{LDH}$ level > $500 \mathrm{U} / \mathrm{L} ; \mathrm{B}$ symptoms. Usage of ChT+Rituximab improved overall survival $(67.6 \%$ vs $42 \%, \mathrm{p}=0.07)$ and reduces the probability of progression of CD20+ B-cell lymphoma ( $8 \%$ vs $37 \%$, p=0.01). Patient's age, ECOG status, disease stage, presence of $\mathrm{B}$ symptoms and International Prognostic Index (IPI) had no impact on the outcome, which indicates to presence of other factors, which could include HIV Status (CD4+ cell levels, and viral load).

\section{Conclusions}

Aggressive DLBCL in HIV-positive patients was diagnosed more often than other lymphoma types. The 2-year OS in patients with HIV and lymphomas was $64 \%$. Adequate ChT, 


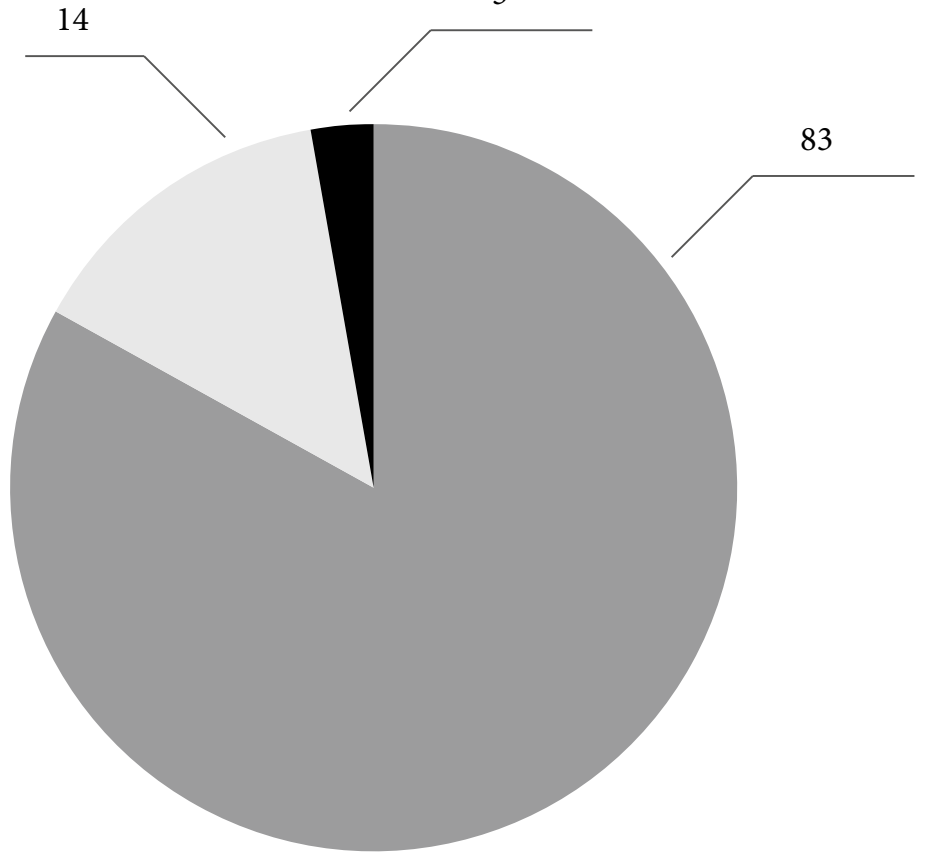

Fig. 1. Histological types of lymphoproliferative diseases in HIV-infected patients

Table 1. Clinical characteristics of the patients under study

\begin{tabular}{|c|c|c|}
\hline \multicolumn{2}{|l|}{ HIV status } & $\%$ pts \\
\hline \multicolumn{2}{|c|}{ HIV before the diagnosis of lymphoma } & $50 \%$ \\
\hline \multicolumn{2}{|c|}{ CD4+ cell and viral load at the diagnosis known } & $40 \%$ \\
\hline \multicolumn{2}{|c|}{ HAART at the moment of lymphoma diagnosis } & $25 \%$ \\
\hline \multicolumn{2}{|c|}{ Co-infection with hepatitis C or B virus } & $42 \%$ \\
\hline \multicolumn{3}{|l|}{ Lymphoma status } \\
\hline \multicolumn{2}{|c|}{ Ann Arbor 4 stage with extra nodular involvements } & $78 \%$ \\
\hline \multicolumn{2}{|c|}{ B-symptoms } & $55 \%$ \\
\hline \multicolumn{2}{|l|}{ ECOG 3-4 } & $17 \%$ \\
\hline \multicolumn{2}{|l|}{ IPI (3>) } & $51 \%$ \\
\hline \multicolumn{2}{|c|}{ One of c-MYC, bcl2, bcl6 tested by FISH } & $8.7 \%$ \\
\hline \multicolumn{2}{|l|}{ EBV tested } & $26 \%$ \\
\hline \multicolumn{3}{|l|}{ Chemotherapy } \\
\hline CT with HAART & of all Ly & $89 \%$ \\
\hline CT with Rituximab & of $\mathrm{CD} 20+\mathrm{Ly}$ & $72 \%$ \\
\hline \multirow[t]{2}{*}{ HL } & BEACOPP & $60 \%$ \\
\hline & ABVD & $40 \%$ \\
\hline \multirow[t]{3}{*}{ NHL } & CHOP & $36 \%$ \\
\hline & EPOCH (SC-/DA-) & $32 \%$ \\
\hline & Hyper-CVAD / BFM & $32 \%$ \\
\hline MM & VAD & $50 \%$ \\
\hline all diagnosis & No CT or steroids & $12.3 \%$ \\
\hline
\end{tabular}


with regard to type and stage of lymphoma in combination with HAART improved overall survival. LDH levels higher than $500 \mathrm{U} / \mathrm{L}$ and B-symptoms were adverse prognostic factors. Usage of Rituximab for CD20 B-cell lymphomas reduced the probability of progression. HIV status seems to play an important prognostic role for the further outcomes. Continuous prospective studies are required in the field.

\section{Keywords}

Lymphoma, HIV, HAART, polychemotherapy, Rituximab, lactate dehydrogenase.

\title{
| Эпидемиология и результаты лечения ВИЧ-ассоциированных лимфом: многоцентровое ретроспективное исследование
}

\begin{abstract}
Марина Попова ${ }^{1}$, Татьяна Шнейдер ${ }^{2}$, Игорь Карягин ${ }^{2}$, Ольга Пономаренко ${ }^{2}$, Зюзгин Илья ${ }^{2,11}$, Ольга Рябыкина Ольга Ружинская ${ }^{2}$, Ольга Успенская ${ }^{2}$, Надежда Медведева ${ }^{3}$, Анна Климович ${ }^{3}$, Всеволод Потапенко ${ }^{3}$, Наталья Котова $^{3,11}$, Елена Зинина ${ }^{4}$, Наталья Попова ${ }^{4}$, Юлия Журба ${ }^{4}$, Александр Мясников ${ }^{5}$, Светлана Мошнина ${ }^{5}$, Алексей Евсеев$^{5}$, Татьяна Поспелова ${ }^{6}$, Камиль Капланов ${ }^{7}$, Татьяна Ксензова ${ }^{8}$, Елена Карягина ${ }^{9}$, Жанна Столыпина ${ }^{9}$ Светлана Дзола $^{10}$, Александр Леванов ${ }^{10}$, Евгения Борзенкова ${ }^{1}$, Анастасия Некрасова ${ }^{1}$, Наталья Михайлова ${ }^{1}$, Людмила Зубаровская $^{1}$, Борис Афанасьев ${ }^{1}$
\end{abstract}

${ }^{1}$ Первый Санкт-Петербургский государственный медицинский университет им. акад. И.П. Павлова, НИИ детской онкологии, гематологии и трансплантологии им. Р.М. Горбачевой, Санкт-Петербург;

${ }^{2}$ Ленинградская областная клиническая больница, отделение онкогематологии №1 и №2, г. Санкт-Петербург; ${ }^{3}$ Городская больница №31, отделение онкогематологии, Санкт-Петербург, Россия;

${ }^{4}$ Сургутская окружная клиническая больница, отделение гематологии, Сургут, Россия;

${ }^{5}$ Республиканская клиническая больница, отделение гематологии, Петрозаводск;

\section{Введение}

Около 70 миллионов людей инфицированы ВИЧ и около 35 миллионов умерло от СПИДа. У пациентов с ВИЧ-инфекцией риск развития злокачественных опухолей, в том числе лимфом, значительно выше, чем в общей популяции. С учетом роста инфицированных ВИЧ, доступности антиретровирусной терапии (АРВТ) увеличивается количество пациентов живущих с ВИЧ и возрастает актуальность проблемы диагностики и лечения лимфом на фоне ВИЧ-инфекции. Цель настоящего исследования оценить эпидемиологию и результаты лечения пациентов с лимфомами на фоне ВИЧ-инфекции, получавших помощь в гематологических стационарах России. Методы. В исследование включено 73 пациента, у которых были диагностированы лимфомы
${ }^{6}$ Гематологический центр, Новосибирск;

${ }^{7}$ Волгоградский областной клинический онкологический диспансер, отделение гематологии, Волгоград;

${ }^{8}$ Областная клиническая больница №1, отделение гематологии, Тюмень;

${ }^{9}$ Городская больница №15 отделение гематологии, Санкт-Петербург;

${ }^{10}$ Клиника профпатологии и гематологии СГМУ, Саратов;

${ }^{11}$ НИИонкологии им.Н.Н.Петрова, химиотерапевтическое отделение онкологии, гематологии и трансплантации костного мозга, Санкт-Петербург

на фоне ВИЧ в 10 гематологических отделениях стационаров Санкт-Петербурга, Сургута, Петрозаводска, Новосибирска, Волгограда, Тюмени и Саратова в период с мая 2006 года по декабрь 2015 года. Проводился анализ данных анамнеза, результатов обследования и лечения в гематологических стационарах и «СПИД-центрах» на основании сложившейся практики. Медиана наблюдения составила 24 (2-110) месяца.

\section{Результаты}

Эпидемиологические характеристики (диагноз) пациентов представлены на рисунке 1. Характеристики ВИЧ и лимфомы и лечения, включенных в исследование пациентов представлены в таблице 1 . 
Общая выживаемость в течение 2х лет у ВИЧ-инфицированных пациентов с лимфомами составила $64 \%$ (ЛХ - 80\%, НХЛ - 60\%, ММ - 100\%), время до прогрессирования - 17\% (ЛХ - 20\%, НХЛ - 16,7\%, ММ - 0\%). Проведение ПХТ в сочетании с АРВТ и назначение ПХТ соответствующей диагнозу и стадии заболевания достоверно улучшает $\mathrm{OB}$, и назначение ПХТ соответствующей диагнозу и стадии заболевания уменьшает вероятность прогрессирования заболевания. Уровень ЛДГ выше $500 \mathrm{U} / 1$ и наличие B-симптомов является прогностически неблагоприятными факторами для ОВ и времени до прогрессирования. Проведение ПХТ в сочетании с ритуксимабом улучшает ОВ $(67,6 \%$ vs $42 \%, \mathrm{p}=0,07)$ и уменьшает вероятность прогрессирования заболевания у пациентов с CD20+ НХЛ (8\% vs 37\%, p=0,01). Возраст пациента, общесоматический статус, стадия заболевания и международный прогностический индекс (IPI) не оказывали влияния на исход лечения пациентов с лимфомами на фоне ВИЧ, что свидетельствует о наличие других факторов, к которым, вероятно, относится ВИЧ-статус (уровень CD4+ клеток и вирусная нагрузка).

\section{Заключение}

У пациентов с ВИЧ чаще диагностируют ДВККЛ, которые характеризуются агрессивным течением. Общая выживаемость пациентов с лимфомами на фоне ВИЧ в течение $2 \mathrm{x}$ лет составляет $64 \%$. Проведение АРВТ в сочетании с ПХТ соответствующей диагнозу и стадии заболевания обеспечивает благоприятный прогноз. Уровень ЛДГ выше 500 U/1 и наличие В-симптомов является прогностически неблагоприятными факторами. Использование Ритуксимаба уменьшает вероятность прогрессирования заболевания у пациентов с CD20+ НХЛ. ВИЧ-статус, по-видимому, играет важную прогностическую роль, для уточнения требуются проспективные исследования.

\section{Ключевые слова}

Лимфомы, ВИЧ, активная антиретровирусная терапия, полихимиотерапия, ритуксимаб, лактатдегидрогеназа. 